\title{
Venous Thromboembolism After Removal of Retrievable Inferior Vena Cava Filters
}

\author{
Takuji Yamagami · Osamu Tanaka $\cdot$ Rika Yoshimatsu • \\ Hiroshi Miura $\cdot$ Tsunehiko Nishimura
}

Received: 25 March 2009/ Accepted: 29 July 2009/Published online: 19 September 2009

(C) The Author(s) 2009. This article is published with open access at Springerlink.com

\begin{abstract}
The purpose of this study was to examine the incidence of new or recurrent venous thromboembolism (VTE) after retrieval of inferior vena cava (IVC) filters and risk factors associated with such recurrence. Between March 2001 and September 2008, at our institution, implanted retrievable vena cava filters were retrieved in 76 patients. The incidence of new or recurrent VTE after retrieval was reviewed and numerous variables were analyzed to assess risk factors for redevelopment of VTE after filter retrieval. In $5(6.6 \%)$ of the 76 patients, redevelopment or worsening of VTE was seen after retrieval of the filter. Three patients $(4.0 \%)$ had recurrent deep venous thrombosis (DVT) in the lower extremities and 2 (2.6\%) had development of pulmonary embolism, resulting in death. Although there was no significant difference in the incidence of new or recurrent VTE related to any risk factor investigated, a tendency for development of VTE after filter retrieval was higher in patients in whom DVT in the lower extremities had been so severe during filter implantation that interventional radiological therapies in addition to traditional anticoagulation therapies were required (40\% in patients with recurrent VTE vs. $23 \%$ in those without VTE; $p=0.5866$ according to Fisher's exact probability test) and in patients in whom DVT remained at the time of filter retrieval $(60 \%$ in patients with recurrent VTE vs. $37 \%$ in those without VTE; $p=0.3637$ ). In conclusion, new or recurrent VTE was rare after retrieval of IVC filters but was most likely to occur in patients who
\end{abstract}

T. Yamagami $(\bowtie) \cdot$ O. Tanaka $\cdot$ R. Yoshimatsu $\cdot$ H. Miura . T. Nishimura

Department of Radiology, Graduate School of Medical Science,

Kyoto Prefectural University of Medicine, 465 Kajii-chyo,

Kawaramachi-Hirokoji, Kamigyo, Kyoto 602-8566, Japan

e-mail: yamagami@koto.kpu-m.ac.jp had severe DVT during filter implantation and/or in patients with a DVT that remained at the time of filter retrieval. We must point out that the fatality rate from $\mathrm{PE}$ after filter removal was high (2.6\%).

Keywords Pulmonary embolism .

Thrombosis of extremities - Interventional procedures . Vena cava filters

\section{Introduction}

Although inferior vena cava (IVC) filter placement is known to be effective in reducing the risk of pulmonary embolism (PE) in selected patients [1, 2], when only shortterm protection is required, ideally a permanent IVC filter would not be placed, considering the long life expectancy of such patients $[2,3]$. Thus, the use of a temporary vena cava filter has found widespread acceptance $[4,5]$. However, problems have been reported, mainly related to the structure of these devices, in that part of the device projects from the insertion site [5-7].

On the contrary, no such problems have arisen in the use of commercially available retrievable filters, which are permanent vena cava filters that can be retrieved when appropriate. Paralleling the increased usage of retrievable vena cava filters in daily clinical practice, reports of their use have increased [8-14]. However, most published reports have focused on the feasibility and safety of withdrawal of the filter [8-15]. On the other hand, there have been few large studies on venous thromboembolism (VTE) after removal of the filter [16]. The purpose of the present study was to evaluate the incidence of VTE (VTE; defined as deep venous thrombosis [DVT] and/or PE) after filter retrieval. 


\section{Subjects and Methods}

Patients

Between February 2001 and July 2008, at our institution, a Gunther Tulip retrievable vena cava filter (GTF) (Cook, Bjaeverskov, Denmark), which is among the commercially available retrievable vena cava filters, was implanted in 163 patients. Removal of the GTF was attempted after temporal implantation in 81 of these 163 patients, with successful retrieval in 76 of the 81 patients. (46 women, 30 men; mean age, 59.3 years; range, $18-87$ years). These 76 patients comprise the cohort of the present study. In this retrospective study, data for analysis were obtained through review of each patient's chart and results of analysis are described according to reporting standards recommended in previous reports $[17,18]$.

Indications for filter placement were the existence of PE and/or DVT in the lower extremities and absolute or relative contraindication for thrombolysis or anticoagulation $(n=30)$, prophylaxis during interventional radiological treatment such as catheter-directed thrombolysis for lowerextremity DVT without PE $(n=18)$, presence of massive PE with DVT in the lower extremities $(n=12)$, prophylaxis in high-risk patients (remote history of DVT without acute DVT or PE and need for surgery; $n=10$ ), and no PE with large, mobile, free-floating thrombi within the IVC $(n=6)$. The distribution of DVT at the time of GTF implantation in these 76 patients is reported in Table 1. Sixteen patients had PE before filter placement. On a general basis, at our institution, filter retrieval was attempted in all cases in which DVT completely or almost completely disappeared after therapy, the remaining DVT was limited to veins under the knee, or PE had disappeared

Table 1 Location of deep venous thrombus in the lower extremities

\begin{tabular}{lc}
\hline Thrombus location & Number \\
\hline IVC & 8 \\
Iliac & 8 \\
Without extension to IVC & 3 \\
With extension to IVC & \\
Iliofemoral & 4 \\
Without extension to IVC & \\
Iliofemopopliteal & 7 \\
Without extension to calf vein & 6 \\
With extension to calf vein & 1 \\
With extension to IVC & 4 \\
Femoral & 2 \\
Femoropopliteal & 25 \\
Popliteal—calf & 8 \\
None &
\end{tabular}

or did not newly develop as revealed on enhanced chest CT.

GTFs were implanted with the intention of retrieval within 10-14 days of the initial implantation, which is the customary period of implantation. When the need to prolong temporary caval filtration greatly exceeded 10 14 days, the period cited in many reports within which a filter can be withdrawn safely [3, 11-13, 19], the first implanted GTF was retrieved and another was inserted near, but not at the same site as, the former GTF $[14,15]$.

After a vena-cavogram was performed to plan the position of filter placement, the GTF was introduced through the right internal jugular or femoral vein and was positioned at the cephalad side of the top of the venous thrombus. The GTF was positioned at the infrarenal IVC in all but seven patients. Of these seven patients, thrombus in the IVC distributed at the level of the renal vein in six, and, in the remaining patient, a huge abdominal tumor compressed the IVC at the infrarenal level, thus the filter was placed in the IVC at the suprarenal level. When introduction of the GTF from the internal jugular vein was difficult or impossible and insertion from the femoral vein had to be avoided because of the existence of a DVT on the approach route, the right subclavian vein was used. The GTF was placed through the sheath introducer according to the manufacturer's instructions. In implanting a GTF, we took care to orient the filter so that the hook pointed away from the contralateral IVC wall, with the open side of the hook directed toward the IVC lumen.

Retrieval was performed using the GTF retrieval set supplied by the manufacturer (Cook). Details of the usual techniques for placement and retrieval of the GTF are described elsewhere $[11,13,15]$. In cases where a trapped thrombus filled one-quarter or more of the height of the filter as observed on venography just before retrieval, the filter was retrieved only after efforts to diminish the clot. Examples of such efforts were manual aspiration of the thrombus using the catheter or sheath introducer inserted from the femoral vein into the inside of the filter and thrombolysis via the catheter introduced into the filter for several days.

All procedures were performed by one of five experienced interventional radiologists at our institution after written informed consent was obtained from each patient. Our institution does not require institutional review board approval for this type of retrospective study. Principles of the Declaration of Helsinki were followed.

As therapy for VTE before retrieval of GTFs, systemic anticoagulation was performed in 68 patients. In eight patients the filter was retrieved without anticoagulation because it was placed temporarily for prophylaxis in highrisk patients undergoing major surgery and VTE did not develop after surgery in these patients. In 18 cases in which 
DVT was so severe as to be resistant to systemic anticoagulation therapy alone, an interventional radiological procedure such as catheter-directed thrombolysis or manual aspiration was added. In all, ultrasound was performed to detect DVT to decide whether the filter could be removed. If sufficient detection of DVT with ultrasound was difficult, enhanced CT images were added $(n=24)$. In all cases with PE $(n=16)$, enhanced chest CT images were obtained before deciding on filter removal. As a result, there was no DVT in the lower extremities in 47 patients as confirmed by ultrasound and/or enhanced CT images, while in 29 patients a small area of chronic thrombophlebitis was present at the time of retrieval of the final filter. In these 29 patients, the recent phlebitis almost completely disappeared and no proximal extension of phlebitis remained as diagnosed by ultrasound and/or enhanced CT images. The PE disappeared in all patients as shown on enhanced chest CT.

\section{Follow-Up}

All patients were observed at the outpatient clinic at our institution. They were scheduled to visit the outpatient clinic every 1-4 months. Differences in the interval between clinic visits were due to individual circumstances of each patient, as this study was performed through analysis of data collected in daily clinical work, not specifically for research purposes. During the follow-up period, hemostasis was monitored by tests that included Ddimer assays. When one or more of the following was observed during follow-up, ultrasound and/or enhanced CT was performed additionally to diagnose VTE every 26 months: (i) VTE remained at the time of filter retrieval, (ii) D-dimer increased to $>0.5 \mu \mathrm{g} / \mathrm{ml}$ during follow-up, (iii) symptoms led the physician to suspect the occurrence of VTE, and (iv) there was a long-term increased risk of VTE (cancer, $n=7$; known hypercoagulable status, $n=2$ ). Warfarin was administered orally when considered necessary.

\section{Investigated Parameters}

The incidence of development or worsening of VTE was investigated. Numerous variables were analyzed to assess risk factors for development or worsening of VTE after retrieval of GTF. Factors investigated were the patient's gender and age, presence of VTE and PE before filter placement, interventional therapies for VTE before filter retrieval, D-dimer at the time of retrieval, period of filter placement, presence of remnant DVT according to ultrasound and/or enhanced CT images at the time of filter retrieval, and administration of anticoagulation therapy after filter retrieval.

\section{Results}

Overall, 109 filters were implanted in 76 patients. The mean period of implantation of the filter was $19.7 \pm 28.6$ (SD) days, ranging from 6 to 264 days. Mean follow-up period after retrieval of the filter was $39.8 \pm 22.1$ months (range, 6-84 months).

In $5(6.6 \%)$ of the 76 patients in whom the filter was retrieved, development or worsening of VTE was seen. Three $(4.0 \%)$ had worsening or recurrence of DVT in the lower extremities that had once completely or almost completely disappeared, and two (2.6\%) had developed PE. The mortality rate during the follow-up period was $2.6 \%$ $(n=2)$; both deaths were due to PE.

Table 2 reports the correlation between recurrent VTE and various risk factors. There were no significant differences in the incidence of redevelopment or worsening of VTE according to any of the risk factors examined. Table 3 reports the characteristics of five patients in whom redevelopment of VTE was observed during follow-up after retrieval of the filter. In cases 3 and 5, PE developed, resulting in death. In cases 1 and 2 DVT in the femoral and popliteal veins redeveloped, and in case 4 DVT redeveloped in the femoral vein. These three patients had another thrombolysis-thromboaspiration procedure combined with insertion of a second retrievable IVC filter; this second IVC filter was left in place for permanent filtration in one patient. In these three cases, DVT disappeared completely or almost completely. In summary, among the five patients with VTE, two died of PE despite having no potential risk factor for VTE, and three had severe thrombophlebitis requiring interventional therapies and also had remnant thrombus present in calf veins.

\section{Discussion}

A multicenter, randomized, open trial of 400 patients with proximal DVT who were at risk for PE was reported by Decousus et al. [2]. Of the 400 patients, 200 were randomized to receive a permanent IVC filter and 200 to receive no filter. PE occurred within the first 12 days after randomization in $1.1 \%$ of patients who received an IVC filter and in $4.8 \%$ of patients who received no filter. These results showed that the rate of occurrence of symptomatic or asymptomatic PE initially was significantly reduced in cases with the implanted permanent vena cava filter compared with those without such a filter. However, after 2 years, there was no difference in recurrence of PE between the two groups (3.4\% with filter vs. $6.3 \%$ without filter). These data suggest that filters may be most beneficial in the short term. At the 2-year follow-up, the rate of recurrent DVT was significantly higher in patients with the 


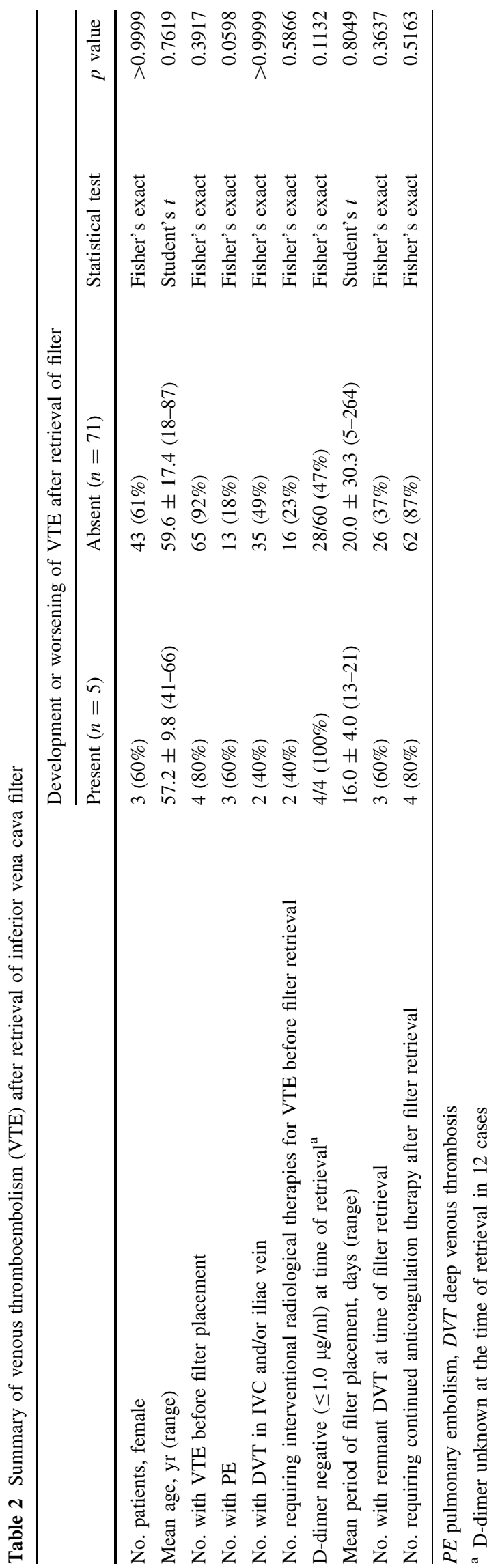

implanted permanent filter than in those without such a filter (20.8\% with filter vs. $11.6 \%$ without filter). Using the same subjects as in the report of Decousus et al. [2], an 8year follow up of patients with permanent vena cava filters also showed a moderate increase in DVT in the filter group ( 35.7 vs. $27.5 \%$ ), while it showed long-term protection by the vena cava filter against embolic recurrence ( 6.2 vs. $15.1 \%$ ) in the filter group [20]. Because their insertion is associated with a significant increase in the occurrence of DVT, the systematic use of permanent vena cava filters in the general population with venous VTE is not recommended [20].

Thus, the use of temporary vena cava filters for a short time found widespread acceptance in previous periods before the use of retrievable filters became widespread $[4,5]$. However, paralleling this increased usage, many reports have described complications related to their insertion. Some were serious and included infection from the part of the device that protruded from the insertion site [5], air embolism through a defective sheath [5], worsening of proximal thrombosis along the attached catheter [6], and migration of the filter into the pulmonary artery [7]. Moreover, many temporary filters require replacement by permanent filters [5] because the maximal implantation period for the device is reached before filtering becomes unnecessary.

Considering these above-mentioned complications and problems with temporary vena cava filters, a retrievable filter that could be implanted without an attached catheter or guidewire and that also could be used as a permanent filter without replacement, if necessary, would offer advantages. The GTF used in the present study was developed relatively recently as one such retrievable vena cava filter. The ease and safety of insertion of the GTF are well known [11-13, 21-23], with some reports citing its use as a nonpermanent filter [3, 11, 12, 21-23]. Millward et al. [22] reported that attempts at retrieval were successful in $98 \%$ of 53 GTFs.

Although the number of reports evaluating retrievability of retrievable vena cava filters has been increasing [10, 15, 22, 23], there have been few reports of follow-up of patients after retrieval $[8,16,22]$. In the report by Millward et al. [22] describing a mean follow-up of 103 days, DVT recurred in $1(2.7 \%)$ of 37 patients in whom the filter was removed. Asch et al. [8] reported that 1 (4.5\%) PE occurred among 22 patients during a postretrieval clinical follow-up of an average of 223 days with Recovery filters. Yavuz et al. [16] reported results of follow-up after retrieval of 72 filters implanted in 67 patients. The mean follow-up period in their study was 20.6 months, and recurrent DVT was seen in $2(2.8 \%)$ patients.

In our study, the rate of recurrent PE was $2.0 \%$ and that of DVT was $4.6 \%$. The incidence of recurrent VTE in our study was similar to that in previous studies, although the 


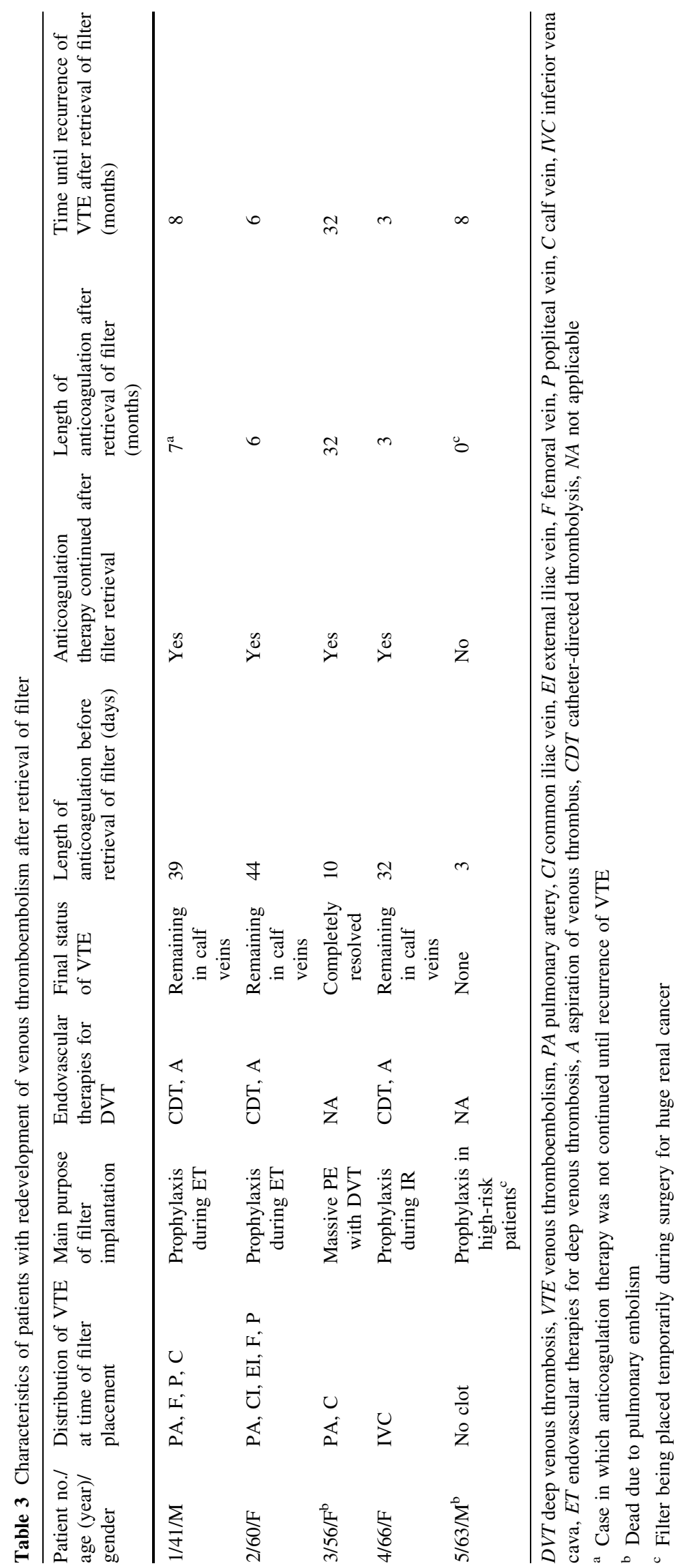


follow-up period was much longer and the number of patients was larger than in the previous reports [8, 16, 22]. In our series, the mean follow-up period after retrieval of the filter was $39.8 \pm 22.1$ months (range, 6-84 months). Such good results might be due to careful long-term observation at the outpatient clinic after filter removal with imaging modalities such as ultrasound and enhanced CT and with blood tests such as D-dimer assays. Among risk factors for recurrent VTE after filter removal, which were not investigated previously to our knowledge, we found that there was a tendency for recurrence of VTE at a higher rate in cases in whom the status of DVT before filter implantation was severe and in whom DVT remained at the time of filter removal. However, the difference was not statistically significant.

However, the fact that two patients died due to recurrent PE in our study suggests the importance of caution regarding indications for filter retrieval and clinical followup after removal, although analysis revealed that they had no particular risk factor except for the interruption of anticoagulation in one of these cases. Considering that retrievable IVC filters are currently used very widely, randomized and prospective studies with large numbers of subjects would be necessary to determine indications for removal of retrievable IVC filters.

Open Access This article is distributed under the terms of the Creative Commons Attribution Noncommercial License which permits any noncommercial use, distribution, and reproduction in any medium, provided the original author(s) and source are credited.

\section{References}

1. Ferris E, McCowan TC, Carver DK et al (1993) Percutaneous inferior vena cava filters: follow-up of seven designs in 320 patients. Radiology 188:851-856

2. Decousus H, Leizorovics A, Parent F et al (1998) A clinical trial of vena caval filters in the prevention of pulmonary embolism in patients with proximal deep-vein thrombosis. N Engl J Med 338:409-415

3. Tay KH, Martin ML, Fry PD et al (2002) Repeated Gunther Tulip inferior vena cava filter repositioning to prolong implantation time. J Vasc Interv Radiol 13:509-512

4. Neuerburg J, Günther RW (1994) Developments in inferior vena cava filters: a European viewpoint. Semin Interv Radiol 11:349357

5. Lorch H, Welger D, Wagner V et al (2000) Current practice of temporary vena cava filter insertion: a multicenter registry. J Vasc Interv Radiol 11:83-88

6. Carcone B, Pernes JM, Carcopino JM et al (1995) Worsening of proximal thrombosis after insertion of a temporary caval filter (3 cases). Rev Med Interne 16:351-353
7. Stosslein F, Altmann E (1998) A rare complication with an Antheor vena cava filter. Cardiovasc Intervent Radiol 21:165-167

8. Asch MR (2002) Initial experience in humans with a new retrievable inferior vena cava filter. Radiology 225:835-844

9. Rosenthal D, Swischuk JL, Cohen SA, Wellons ED (2005) OptEase retrievable inferior vena cava filter: initial multicenter experience. Vascular 13:286-289

10. Oliva VL, Szatmari F, Giroux MF, Klemming BK, Cohen SA, Soulez G (2005) The Jonas study: evaluation of the retrievability of the Cordis OptEase inferior vena cava filter. J Vasc Interv Radiol 16:1439-1445

11. Neuerburg JM, Gunther RW, Vorwerk D et al (1997) Results of a multicenter study of the retrievable Tulip vena cava filter: early clinical experience. Cardiovasc Intervent Radiol 20:10-16

12. Millward SF, Bhargava A, Aquino J Jr et al (2000) Gunther Tulip filter: preliminary clinical experience with retrieval. J Vasc Interv Radiol 11:75-82

13. Millward SF (2001) Gunther Tulip retrievable filter: why, when and how? Can Assoc Radiol J 52:188-192

14. Yamagami T, Kato T, Iida S et al (2005) Gunther Tulip inferior vena cava filter placement during treatment for deep venous thrombosis of the lower extremity. Cardiovasc Intervent Radiol 28:442-453

15. Yamagami T, Kato T, Hirota T, Yoshimatsu R, Matsumoto T, Nishimura T (2007) Evaluation of retrievability of the Gunther Tulip vena cava filter. Cardiovasc Intervent Radiol 30:226-231

16. Yavuz K, Geyik S, Hoppe H, Kolbeck KJ, Kaufman JA (2008) Venous thromboembolism after retrieval of inferior vena cava filters. J Vasc Interv Radiol 19:504-508

17. Greenfield LJ, Rutherford RB (1999) Recommended reporting standards for vena caval filter placement and patients follow-up. J Vasc Interv Radiol 10:1013-1019

18. Millward SF, Grassi CJ, Kinney TB et al (2005) Reporting standards for inferior vena caval filter placement and patient follow-up: supplement for temporary and retrievable/optional filters. J Vasc Interv Radiol 16:441-443

19. de Gregorio MA, Gamboa P, Gimeno MJ et al (2003) The Gunther Tulip retrievable filter: prolonged temporary filtration by repositioning within the inferior vena cava. J Vasc Interv Radiol 14:1259-1265

20. The PREPIC Study Group (2005) Eight-year follow up of patients with permanent vena cava filters in the prevention of pulmonary embolism. The PREPIC randomized study. Circulation 112:416-442

21. Yamagami T, Kato T, Iida S et al (2003) Retrievable vena cava filter placement during treatment for deep venous thrombosis. $\mathrm{Br}$ J Radiol 76:712-718

22. Millward SF, Oliva VL, Bell SD et al (2001) Gunther Tulip retrievable vena cava filter: results from the registry of the Canadian interventional radiology association. J Vasc Interv Radiol 12:1053-1058

23. Yamagami T, Kato T, Hirota $\mathrm{T}$ et al (2006) Prophylactic implantation of inferior vena cava filter during interventional radiological treatment for deep venous thrombosis of the lower extremity. Br J Radiol 79:584-591 